\title{
Electrical connection network within an electrically conductive adhesive
}

\author{
D.Busek, P. Mach \\ Department of Electrotechnology, Faculty of Electrical Engineering \\ Technická 2, 16627 Prague, Czech Republic, Phone: +420 224352355 \\ busekd1@fel.cvut.cz
}

\begin{abstract}
This paper deals with the connection network in isotropically conductive adhesives (ICAs). The reader will learn about conductive network creation, tunneling effect, possible ways of modeling the conductive network and differences between failures within soldered joints and electrically conductive adhesive joints. This article compares theoretical models with real measurement and properties of the electrically conductive adhesive. The inner structure of the adhesive that is either formed by micro-sized particles, or micro-sized particles mixed with nano-sized particles, is described.
\end{abstract}

\section{INTRODUCTION}

The soldering process is being widespread used in the world for past few decades. In July 2006, the use of several chemicals and agents was in the European Union restricted according to the RoHS (Restriction of hazardous substances) directive. Among these substances is also lead that is commonly used in solders. The demand that devices supplied to the commercial market cannot contain lead generated an industry-wide effort to identify lead free alternatives.

One of the ways is to use electrically conductive adhesives (ECAs). These contain filler and binder (silver balls/flakes and an epoxy resin). Another major reason for the interest in ECAs is the requirement of increasing miniaturization. With solders, very fine pitch is not possible as the solder would create short circuits. For ultra-fine pitch applications (up to $50 \mu \mathrm{m}$ spacing), anisotropically conductive adhesives are most suitable [1].

\section{Motivation}

Dominant mean for flat panel displays (LCD's) are just the above mentioned anisotropically conductive adhesives. Some reliability issues are major obstacles that prevent even wider application of the conductive adhesives. These include worsening parameters after fatigue, impact, or exposure to harsh environmental conditions. To improve the performance of conductive adhesives for electronic applications and to use them as a solder replacement, fundamental studies are necessary to develop a better understanding of the mechanisms underlying these reliability problems.

Knowing and understanding the connection network inside a conductive joint allows more precise lifetime prediction of a joint, reveals its weaknesses and offers the opportunity to remove or suppress them. In previous years, several measurements with different types of substrates, different straining and different conductive adhesives were undertaken. We tested the ability of the adhesives to withstand atmospheric or mechanical stress by measuring electrical properties before and after the applied stress. Understanding the principles of the real connection network inside the adhesive conducts may clear up the question why the increase of amont of conductive paths by the addition of nanoparticles does not always improve resistance of the conductive material or why the strength of a joint gets significantly higher.

\section{CONDUCTION PRINCIPLE}

The electrical conduction of conductive adhesives is provided by the connections of filler particles. The most used filler is pure silver. In addition to silver, other metals (i.e. nickel) are also sometimes used. The major reason for the use of silver is that even $\mathrm{Ag}$ oxides are well conductive. When the volume fraction of conductive particles is higher than the percolation threshold, the conductive particles contact with each other and form a conductive network. Since the bulk resistance of the filler particles themselves is very 
small (silver has best conductivity among metals), the contact resistance is the major contributor of the resistance of the adhesive. The geometric properties of the filler also significantly influence the electrical conduction. Most companies use rather silver flakes instead of spherical fillers, not just because their manufacturing is easier and therefore cheaper, but also the mathematical modeling shows that lower resistivity can be achieved using rectangular or elliptically shaped filler as flakes. [3]

\subsection{Tunnel resistance}

Tunnel resistance is another type of resistance on the contact surface. Even if an insulating thin film on the conductive particle is present, the electron can penetrate this barrier, even if its energy is lower than the barrier. This effect can be described using quantum physics, where the electron is considered to be a wave. See the illustrative picture below [2]:

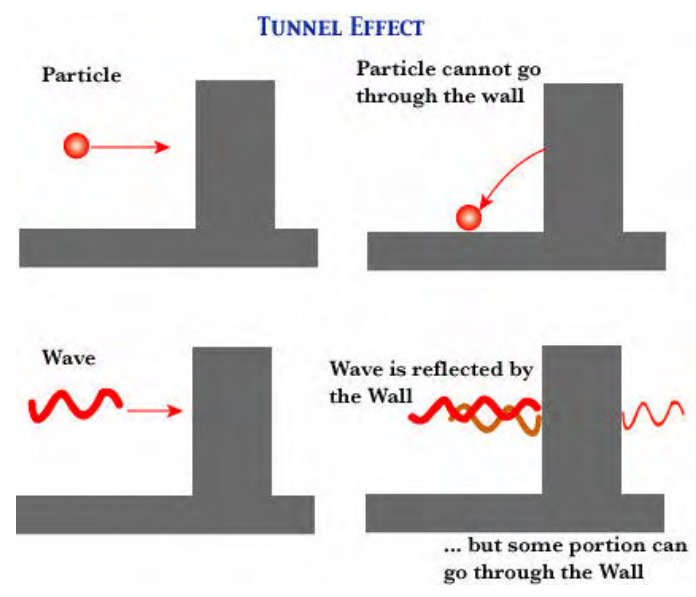

Figure 1. Tunnel effect.

The barrier can be insulating contaminant, air film or a very small adhesive-filled gap between conductive particles. Tunnel resistivity $\sigma$ has the unit of $\Omega . \mathrm{m}^{2}$ (resistance per $\mathrm{m}^{2}$ of the film). The tunnel resistance also depends strongly on the thickness of the film and chemical properties of the film.

$$
\mathrm{R}_{\mathrm{t}}=\sigma /\left(\pi \cdot \mathrm{a}^{2}\right)
$$

where the contact surface is circular with radius $a$

The contact resistance determines at the end the total resistivity of the adhesive material. The higher the contact force in the adhesive (due to shrinkage of the epoxy resin) exists, the fewer barriers need to be tunneled and the lower the adhesive resistance is.

\subsection{Constriction resistance}

If two contact particles are made of the same material and have a bulk resistivity of $\rho$, and the radius of the circular conducting area among them is $a$, then the constriction resistance is:

$$
R_{c}=\rho / 2 a
$$

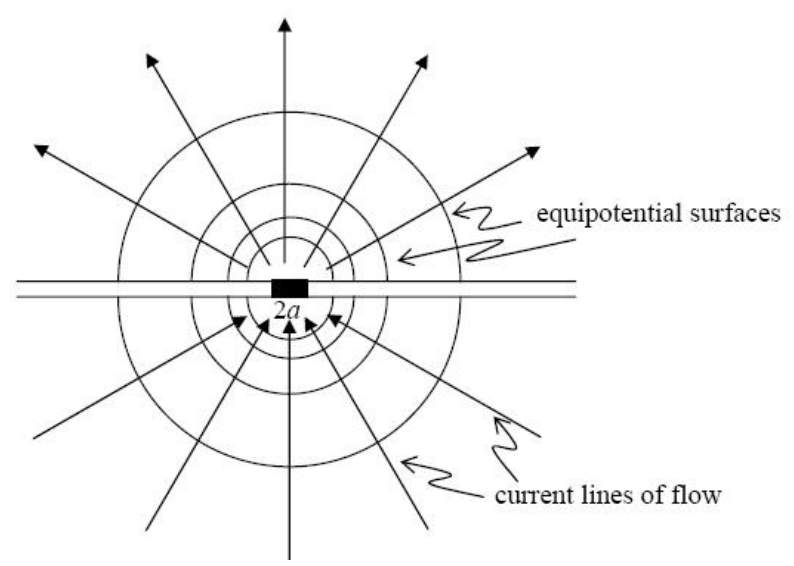

Figure 2. Contact of two semi-infinite members.

The total contact resistance on a contact interface is the sum of the constriction resistance and tunnel resistance. If the contact area is circular with a radius $a$, the contact resistance can be expressed as

$$
\mathrm{R}=\mathrm{R}_{\mathrm{t}}+\mathrm{R}_{\mathrm{c}}=\rho / 2+\sigma /\left(\pi \cdot \mathrm{a}^{2}\right)
$$

\subsection{Adhesive and solder evaluation}

Different evaluation of the quality of the joint needs to be used when conductive adhesive instead of solder is present. While the soldered joint keeps conducting until being physically disconnected (crack must go through the whole joint), the conductive adhesive could fail electronically well before any mechanical failure appears. Therefore the fatigue should be considered in terms of deterioration of electrical conduction rather than any mechanical adhesion or breakage [3]. 


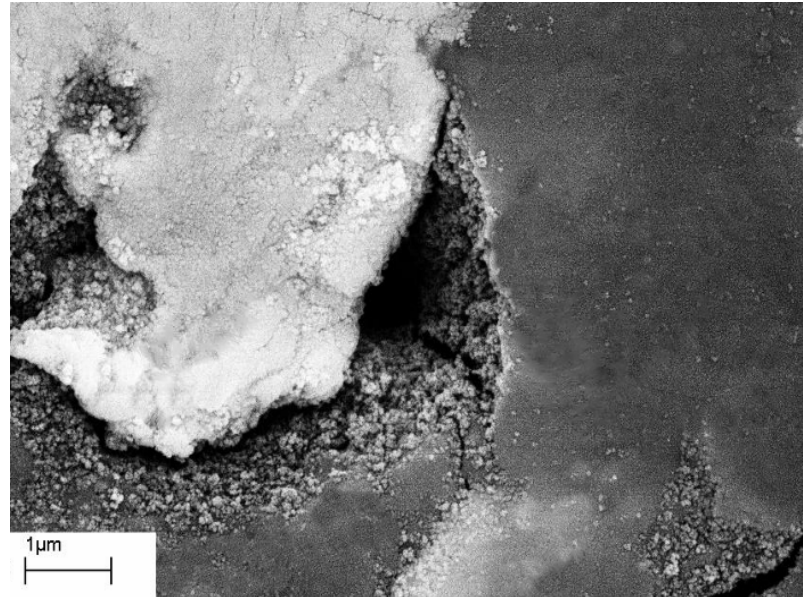

Figure 3. Silver microparticle falls off the epoxy resin after fatigue is applied.

The above picture shows micro sized particle freed from the epoxy resin after fatigue. While the surrounding epoxy stays intact, the electrical connection is gone.

\section{ACCELERATED TESTS}

Accelerated tests are used to simulate the long impact of the surrounding conditions for the device. In laboratory conditions the device or observed part is put into a chamber with stressing conditions. This can be for example atmosphere with $85 \% \mathrm{RH}$ at $85^{\circ} \mathrm{C}$ for 100 hours (damp-heat testing), thermal cycling or raised temperature $\left(125^{\circ} \mathrm{C}\right)$. In past years, we conducted accelerated testing of different conductive adhesives on different substrates where one of the results was that HAL or nickel finish is not suitable as a substrate for epoxy based conductive adhesives. [4]

Accelerated tests revealed that the substrate type influence is of no lower significance compared to the structure of the adhesive. Moisture showed to be most influencing factor. In order to lower its negative influence, corrosion inhibitors can be added to the adhesive according to $\mathrm{Li}$ at al. [5]

\section{Preferred Filler Particle Size}

An easy answer of how big the particles in the adhesive should be in order to get the best properties unfortunately does not exist.

Bin Su [3] conducted in his work mathematical modeling with the result that "smaller particles are preferred if one wants to get a smaller resistivity". This modeling included conduction network with ball fillers with diameter ranging from $2 \mu \mathrm{m}$ to $8 \mu \mathrm{m}$ and the volume fraction of conductive particles being $30 \%$ (that corresponds to a weight fraction of approximately $80 \%$ ). Even though this may work for microparticles of that size, when nanoparticles are used, this approach will not work as nanoparticles agglomerates and may not fulfill the condition that the filler is uniformly distributed in the resin. An agglomerate having the same size as a micro-particle can not exhibit comparable resistance to one particle as many constriction and tunneling resistances are present within such agglomerate. This approach can reveal why in our experiments, the resistance slightly raised after $4 \%$ nanoparticles were added to the microparticle ECA (see Figure 6 and Figure 7 below).

The mix-up of particles of two sizes could theoretically greatly improve the conduction of conductive adhesives. This can be explained intuitively: smaller particles fill in the gaps between larger particles (see Figure4, left picture and Figure5) and more conduction paths are created. The fact that most commercial conductive adhesives manufacturers use flakes of difference sizes to compound conductive adhesives confirms this.

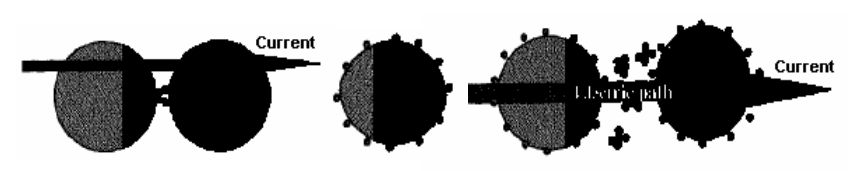

Figure 4. Conductive adhesive with small nanoparticle addition (left), moderate (middle) and too high addition (right)

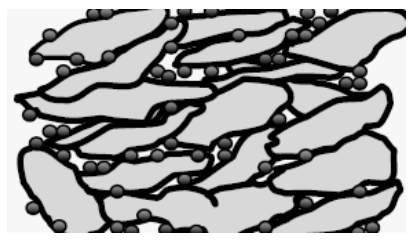

Figure 5. Nanoparticles can fill gaps between microsized particles

In order to prove that mathematical models works in real life, standard ECA containing only microparticles 3-6 $\mu \mathrm{m}$ in diameter was provided with small addition of nanoparticle silver (nanoparticle diameter approx. $100 \mu \mathrm{m}$ ). The original ECA is in the figures named as S0. S1 and S3 are both modified adhesives, where S1 contained $2 \%$ "nanosilver" and S3 $4 \%$. The resistance values did not decline as expected at this moment. But the difference was very 
clearly visible after the samples were exposed to mechanical straining. (see Figure 7). The nanoparticle addition significantly improved resistance to mechanical stress.

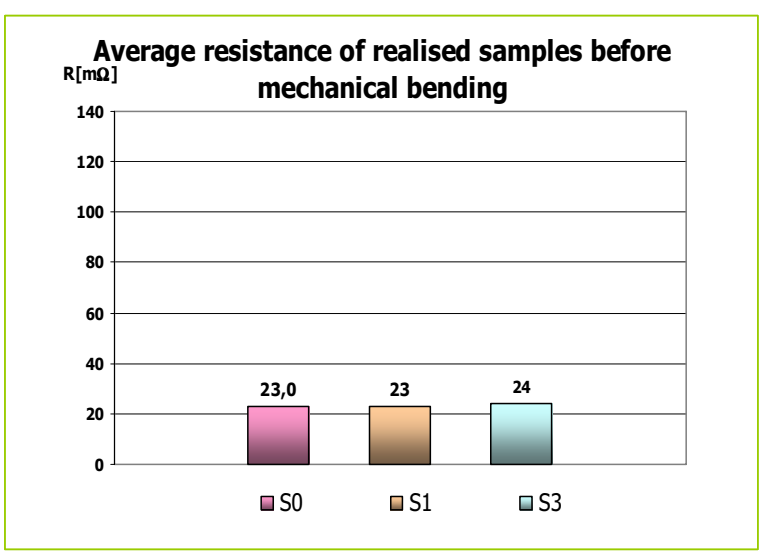

Figure 6. S0 (left column) is standard ECA, other two contain nanoparticles (without stress)

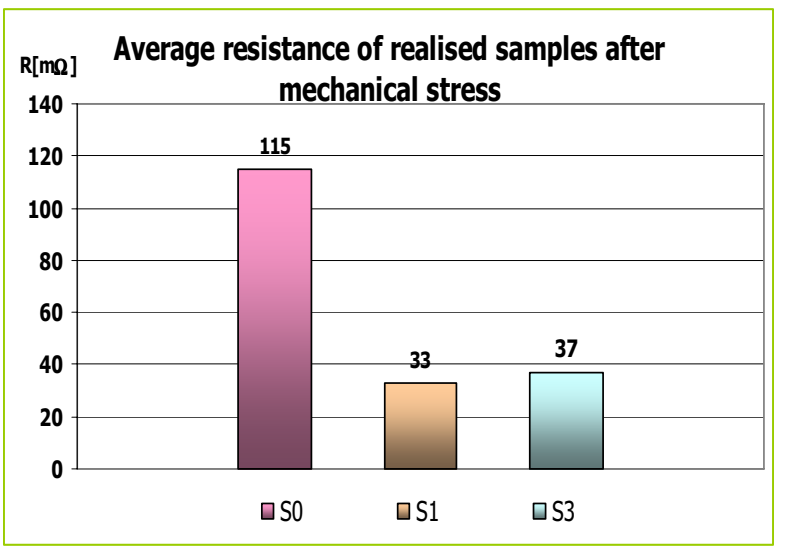

Figure 7. Adhesive without nanoparticles is not stable after mechanical straining (S0). ECAs with nanoparticles are stable (after mechanical stress)

It is difficult to explain the reason for such a big improvement. Further research is necessary here.

\subsection{Particle filler shape}

Another important parameter in electrically conductive adhesives is so called effective volume fraction. Not all filler particles take part in the current conduction. (That may be the reason for the mechanical resistance improvement in our research and no resistivity change.) The effective volume fraction is defined as the volume fraction of the particles that contribute to the electrical conduction in conductive adhesives. It is calculated by dividing the volume of the particles that are in the resistor network by the total volume of the conductive adhesive. The following picture shows this clearly (see Figure8) [6]

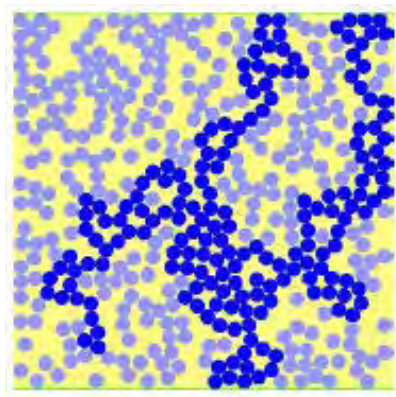

(a), filling factor $\phi=54.5 \%$; aspect ratio $X=1$.

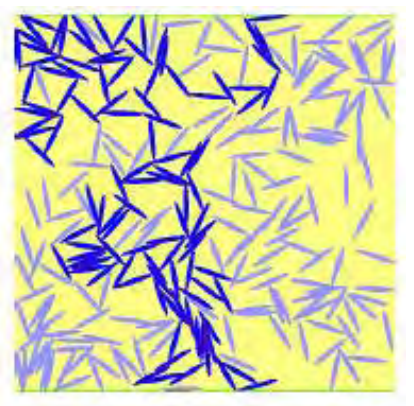

(b) filling factor $\phi=30.5 \%$; aspect ratio $X=8$.
Figure 8. Particle arrangements with different filling factor and aspect ratio each for a percolation probability of $50 \%$

(all particles having the same area)

With non-spherical filler (aspect ratio $\neq 1$ ), the filling factor can be much lower while the percolation threshold stays the same. Flake particles have bigger effective volume fractions than spherical particles. However, smaller effective volume fraction doesn't always give bigger resistivity. For example, spherical particles of two sizes have a small effective volume fraction but still give a small resistivity, which means that mixing particles of two sizes could form the conduction network in conductive adhesives more efficiently. Also, silver nanorods with even higher aspect ratio (length divided by thickness) or nanowire usage could lead to even higher material saving. The disadvantage may be lower maximal transferred current due to very thin conductive path. On the other hand, ECA's were never intended for high current applications.

\section{Conclusion}

The overview of network creation was presented. Performed experiments proved that even though modern simulations can be performed to estimate the optimal size and shape of the nanofillers, experimental measurement needs to be done as well. Our previous research [4] showed that nanoparticles influence the overall parameters significantly even in small addition. The most obvious was the viscosity change the smaller the particles were, the more viscous the adhesive was. Adhesives filled only with nanoparticles can probably achieve superior 
conductivity, but the viscosity does not allow us to fill the adhesive with $30 \%$ of the nanofiller in volume.

There will always be the possibility to improve the adhesives furthermore and understanding the conductive network creation and principles within the adhesive just allows researchers to make more and more improvements.

This research was supported by grant: Czech Republic - MSM No.6840770021 - Diagnostic of Materials

\section{REFERENCES}

[1] Johan Liu, Zonghe Lai: Reliability of Anisotropically Conductive Adhesive Joints on a Flip-Chip/FR-4 Substrate, in: Journal of Electronic Packaging -September 2002 -- Volume 124, Issue 3, pp. 240-245

[2] Kyushu University: http://www.bun.kyotou.ac.jp/ suchii/Bohr/tunnel.html
[3] Bin Su: Electrical, thermomechanical and reliability modeling of electrically conductive adhesives, School of Mechanical Engineering, Georgia Institute of Technology, May 2006

[4] Bušek, D. - Mach, P.: Properties of Joint Made Using Electrically Conductive Adhesives, In: Applied Electronics 2005 - International Conference Pilsen. Pilsen: University of West Bohemia, 2005, s. 49-52. ISBN 80-7043-369-8.

[5] Li, L., et al. Reliability and failure mechanism of isotropically conductive adhesives joints. In Proceedings of the 1995 45th Electronic Components \& Technology; Conference, May 21-24 1995. 1995. Las Vegas, NV, USA: IEEE, Piscataway, NJ, USA.

[6] M. Mündlein, J. Nicolics: Electrical Resistance Modeling of Isotropically Conductive Adhesive Joints, Institute of Sensor and Actuator Systems, Vienna University of Technology 